\title{
LA TECHNOLOGIE DES RÉSEAUX SOCIAUX ET LA GESTION DES PERSONNES DANS LE RECRUTEMENT ET LA SÉLECTION DE SES COLLABORATEURS
}

\section{ARTICLE ORIGINAL}

SUMAR, Ramiro Rodrigues ${ }^{1}$

SUMAR, Ramiro Rodrigues. La technologie des réseaux sociaux et la gestion des personnes dans le recrutement et la sélection de ses collaborateurs. Revista Científica Multidisciplinar Núcleo do Conhecimento. An 06, Ed. 07, Vol. 12, p. 74 à 90. Juillet 2021. ISSN : 2448-0959, Lien d'accès: https://www.nucleodoconhecimento.com.br/administration-des-

affaires/collaborateurs, DOI: 10.32749/nucleodoconhecimento.com.br/administrationdes-affaires/collaborateurs

\section{RÉSUMÉ}

Objectif : Décrire l'impact que les réseaux sociaux peuvent avoir sur le recrutement et la sélection de leurs employés. Question Problème : Comment le réseau social peut-il favoriser le recrutement et la sélection des collaborateurs d'une entreprise ? Méthodologie : Analyse documentaire. Résultats : Les données probantes sur les résultats ont montré que les technologies par l'entremise des réseaux sociaux peuvent être pertinentes pour le recrutement et la sélection des personnes pour l'organisation. Mais ce recrutement doit se faire avec un regard différencié sur chaque type de réseau social par le recruteur. Considérations finales : Le recrutement et la sélection ont évolué en tant que mode traditionnel (face à face) pour le mode technologique (virtuel). L'étude mentionne que les réseaux sociaux sont des outils capables d'apporter au recruteur des candidats capables de prendre

\footnotetext{
${ }^{1}$ Maîtrise en contrôle, spécialiste en gestion de la technologie, administration stratégique, sécurité de I'information et diplômé en sciences comptables.
}

RC: 93822

Disponível em: https://www.nucleodoconhecimento.com.br/administration-des- 
l'organisation de manière responsable et qu'il n'y a pas d'obstacles dans le monde virtuel pour trouver le candidat idéal. II est souligné l'importance d'étendre cette étude basée sur des preuves scientifiques, dans laquelle des recherches peuvent être menées dans les entreprises pour l'utilisation des réseaux sociaux dans le suivi de leurs employés.

Mots-clés : Gestion des personnes, Réseaux sociaux, Technologies de l'information et de la communication.

\section{INTRODUCTION}

Les entreprises ont dans leurs principaux objectifs d'obtenir des résultats, cependant, pour que cela se produise, la première étape consiste à embaucher des professionnels qualifiés et compétents pour faire partie de l'équipe. Parmi les départements qui composent une entreprise, il y a la partie de la gestion des ressources humaines, qui a pour fonction de recruter pour l'organisation son personnel professionnel (CARVALHO, 2015).

Le mot "Gérer » consiste en le processus de prise de décision et la réalisation d'actions (qu'elles soient collaboratives, pour améliorer les ressources, l'interaction, entre autres) afin que les objectifs souhaités par l'entreprise puissent être atteints (COSTA, 2018). Par conséquent, l'accent de la gestion des ressources humaines (ou gestion des personnes) est lié à la performance des compétences, parmi celles-ci: l'efficience, l'efficacité, la productivité, la satisfaction et l'engagement envers les personnes, la survie de l'organisation, la qualité, la compétitivité et la responsabilité sociale (SPADER, 2019).

Le manager doit avoir plusieurs qualités pour pouvoir lister son entreprise, parmi lesquelles le leadership, la responsabilité et l'empathie pour arbitrer la performance de ses collaborateurs, pour cela il a besoin de savoir écouter et être ouvert aux suggestions, être participatif, et s'impliquer, principalement, dans les tâches

RC: 93822

Disponível em: https://www.nucleodoconhecimento.com.br/administration-des- 
cherchant à être complice de la délégation de ceux-ci (DE BEM NORO; STUKER; DE OLIVEIRA, 2015).

Selon Ribeiro (2017), le gestionnaire des ressources humaines ou le gestionnaire des personnes, en plus d'être qualifié pour le poste, doit avoir des outils (évaluation du rendement; rétroaction continue; technologies de l'information et de la communication, entre autres), nécessaires à la réalisation d'une bonne administration, étant donné que la partie ressources humaines est l'endroit où divers processus seront exécutés afin que l'entreprise puisse garantir son nom sur le marché des affaires, processus qui visent à recruter des employés pour compléter le système organisationnel.

Ce recrutement se fait sous plusieurs formes, la plus utilisée aujourd'hui est à travers les technologies. L'ère technologique pour les organisations vient avec l'objectif d'améliorer la qualité du service en apportant la vitesse dans le développement des produits / services, la fiabilité des données d'entreprise, une plus grande performance par les employés entre autres qualités et, assurant plus de sécurité à ses utilisateurs. Dans la gestion des personnes, les technologies favorisent une plus grande portée des personnes à recruter, en plus d'aider le gestionnaire dans le moment de connaître vraiment la vie personnelle de ses employés (SILVA, 2019; PORTO et al., 2016; SANTOS, 2018).

Selon Motta et al. (2017), le réseau mondial d'ordinateurs (Internet) favorise que les relations entre les employés et les gestionnaires peuvent construire de nouvelles cultures de relation et de connaissance, qui peuvent résulter des connaissances et des informations dérivées de l'accès à Internet, avec plus d'intensité des réseaux sociaux accessibles ou non dans l'environnement de travail.

Il est essentiel de comprendre que la gestion des personnes a joué un rôle important au sein des organisations. Ce rôle est calqué non seulement sur les politiques et les pratiques, mais cherche également à mettre en évidence dans les facteurs

RC: 93822

Disponível em: https://www.nucleodoconhecimento.com.br/administration-des- 
organisationnels des problèmes liés tels que: les tensions entre les travailleurs, une mauvaise organisation au sein de l'environnement de travail, des problèmes culturels caractérisant les préjugés, afin de pouvoir contrôler les dommages causés par un éventuel harcèlement moral et qui affecteront l'ensemble de l'organisation de l'entreprise (LEAL; MATOS; FONTANA, 2013).

L'étude en question met en avant l'importance des technologies pour le recrutement et la sélection des employés qui cherchent à travailler dans une organisation. La justification de cette étude vient du chercheur ayant de l'expérience dans les technologies de l'information et de la communication et observant que dans les réseaux sociaux l'offre d'emploi se pose avec une demande très importante, ainsi que l'observation de la façon dont l'entretien sera organisé (en particulier les personnes de niveau supérieur l'entretien a lieu par google meet). Dans ce segment, la question problématique de cette étude est : Comment le réseau social peut-il favoriser le recrutement et la sélection des salariés d'une entreprise?

Par conséquent, la principale contribution à la réalisation de cette étude est basée sur les connaissances sur l'utilisation des technologies par les gestionnaires de personnes, des réseaux sociaux, ce qui rend pertinent d'ajouter plus de connaissances à la fois au professionnel et à la société en général. Pour la communauté scientifique, la pertinence de l'étude s'ajoute à l'élargissement des connaissances sur l'utilisation des technologies pour le recrutement et la sélection des personnes.

L'objectif proposé dans cette étude était de décrire l'impact que les réseaux sociaux peuvent avoir sur le recrutement et la sélection de leurs employés.

Pour que cette étude ait lieu, nous avons recherché une base théorique liée aux thèmes suivants: Recrutement et sélection dans l'entreprise; les technologies de l'information ayant une incidence sur la gestion des personnes; Réseaux sociaux pour le recrutement et la sélection d'une entreprise.

$\mathrm{RC}: 93822$

Disponível em: https://www.nucleodoconhecimento.com.br/administration-des- 
Sur la base de cette base théorique, la méthodologie de la conception de l'étude est basée sur la revue bibliographique.

\section{RÉFÉRENCE THÉORIQUE}

\subsection{LE RÔLE DU RECRUTEMENT ET DE LA SÉLECTION DANS L'ENTREPRISE}

En parlant de recrutement et de sélection des personnes, Oliveira et Bueno (2018) décrivent que ce système de choix vient des hommes des cavernes, où les choix des gens de développer les attributions imposées par le système social de l'époque ont été donnés par leur force, étant la condition la plus forte pour participer aux chasses pour l'alimentation de leur peuple. Dans le contexte actuel, cette sélection devient de plus en plus difficile.

Chiavenato (2002) mentionne l'importance du recrutement et de la sélection pour l'organisation. L'auteur souligne que les deux outils de choix organisationnel sont directement liés au développement de l'entreprise et, même ayant des attributions différentes, ont le même objectif : identifier l'employé approprié pour constituer l'organisation.

Le recrutement peut être classé comme interne ou externe. Le recrutement étant interne se produit lorsque l'organisation apporte un changement à son personnel. Ce changement résulte de la performance du travailleur dans son secteur et du besoin de l'entreprise, ce qui permet le choix de ce travailleur observé depuis un certain temps, de la promotion, c'est-à-dire de leur transfert vers des départements ou des postes plus importants. De cette modification, cet employé est formé, afin que sa performance puisse obtenir de meilleurs résultats au sein de l'entreprise avec ses activités (CANALLI; DE ANDRADE, 2016).

$\mathrm{RC}: 93822$

Disponível em: https://www.nucleodoconhecimento.com.br/administration-des- 
Lors du recrutement externe, l'employé peut être choisi par des processus ou par d'autres moyens. Les exemples en relation avec ces médias sont les annonces dans la presse, les conférences dans les écoles et les universités, la diffusion dans les associations de classe, par des recommandations de tiers, les agences pour l'emploi, les dossiers de candidats stockés dans l'entreprise qui se sont déjà présentés et l'échange d'entreprises. Dans le recrutement externe, les annonces de l'entreprise et par des pages de diffusion des offres d'emploi sur Internet apportent cette notoriété que les employés sont nécessaires (SILVA; FERRETT; MANCINI, 2014).

Par conséquent, tous les efforts de l'entreprise pour amener dans son cercle de nouveaux employés sont liés au recrutement. Alberton (2002) décrit que le processus de recrutement est considéré comme l'une des étapes les plus importantes du processus d'admission d'un nouveau professionnel. L'auteur souligne que la plupart des entreprises recherchent au premier plan dans l'environnement interne, puis se tournent vers le marché du travail.

Mozer (2016) souligne que l'organisation a des cultures différentes les unes des autres. Ces cultures peuvent faire en sorte que le travail soit fait de différentes manières. Cependant, le recrutement et la sélection ne sont pas différents. Le processus par lequel le recrutement de l'organisation est effectué a ses variations, mais les moyens utilisés sont les mêmes et peuvent être utilisés par le biais des technologies de l'information et de la communication, où les postes vacants sont divulgués pour les postes au sein de l'entreprise. Dans le cas des sélections, il y a une décision par laquelle le candidat se positionnera du meilleur rendement au poste qui lui est offert.

Le recrutement interne ou externe présente des avantages et des inconvénients. Pour l'organisation ainsi que pour les concurrents au poste mis à disposition dans l'entreprise, ces conditions doivent être examinées afin d'identifier la meilleure façon

$\mathrm{RC}: 93822$

Disponível em: https://www.nucleodoconhecimento.com.br/administration-des- 
de recruter. Les figures 1 et 2 font état de ces avantages et inconvénients du recrutement interne et externe.

Figure 1 : Avantages du recrutement interne et externe

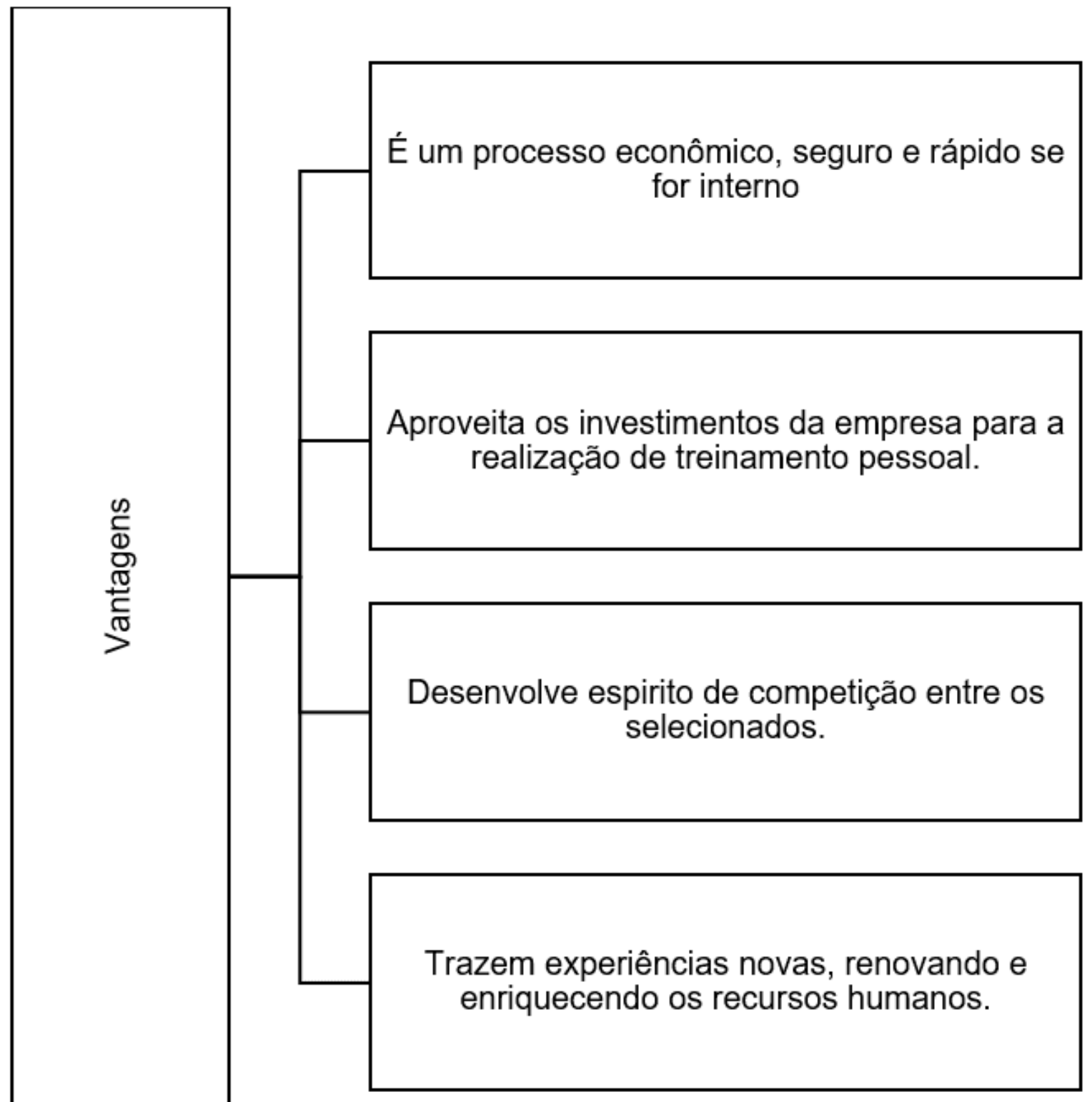

Source : Tiré de Chiavenato (2002).

RC: 93822

Disponível em: https://www.nucleodoconhecimento.com.br/administration-des- 
Figure 2 : Inconvénients du recrutement interne et externe

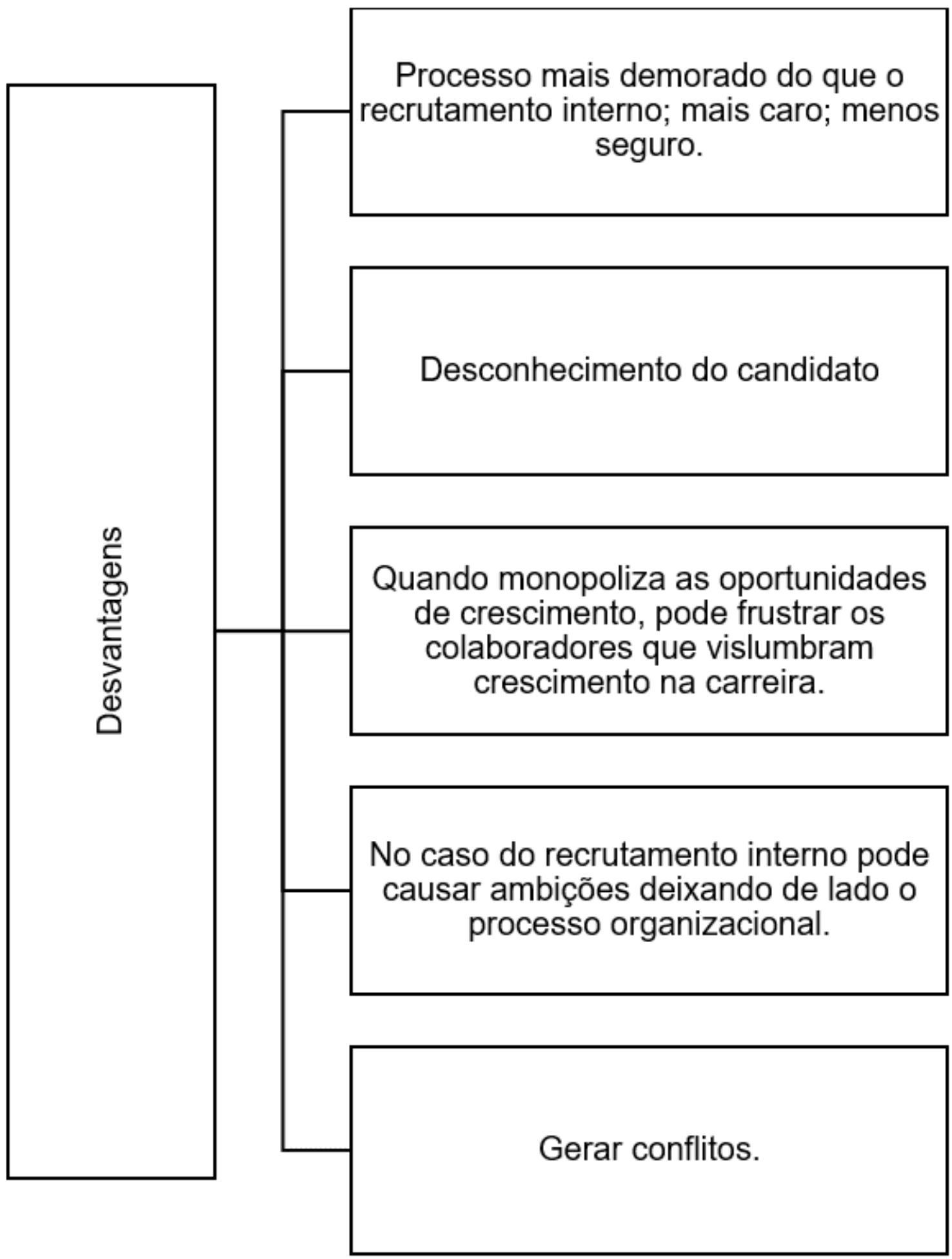

Source : Tiré de Chiavenato (2002).

RC: 93822

Disponível em: https://www.nucleodoconhecimento.com.br/administration-des- 
Par conséquent, le recrutement et la sélection sont des outils dans lesquels les entreprises sont prêtes à investir, ajoutant de la valeur aux entrepreneurs. Ainsi, les employés sélectionnés doivent être en mesure d'exercer la fonction pour laquelle le poste vacant a concouru et connaître les politiques que l'entreprise adopte.

Chiavenato (2002) affirme que ces outils peuvent être utilisés en parallèle. Le choix de la manière dont il devrait être utilisé dépendra de la situation, du nombre de candidats, du profil souhaité et, surtout, des exigences nécessaires à l'exercice des fonctions.

II est à noter que tous les environnements ne fournissent pas les conditions pour former des leaders, cette action développée par la gestion des personnes rend possible le recrutement interne grâce au leadership, que l'employé a l'autonomie et la prise de décision devant l'autre équipe, harmonisant ainsi l'environnement, le laissant adéquat et principalement capable d'augmenter la rentabilité et la productivité des autres (LEITE, 2019).

\subsection{OPTIMISATION DE LA GESTION DES PERSONNES PAR GOUVERNANCE INFORMATIQUE}

La gestion des personnes au fil des ans a subi des adaptations résultant de transformations profondes qui peuvent impliquer la mise en œuvre d'actions pour obtenir des résultats. Selon Wanzerller et al. (2018), l'origine du nom management vient du latin "gestione ", qui définit son sens comme un acte ou un effet de gestion ou de gestion ou de gestion et de personne du latin « persona » qui désigne un homme ou une femme.

Depuis les années 1980, la mondialisation a pris le dessus sur le scénario économique, avec l'évolution des communications, du développement technologique et de la compétitivité. Cet avènement technologique a conduit plusieurs entreprises à acquérir de nouvelles connaissances sur les technologies de l'information dans les

RC: 93822

Disponível em: https://www.nucleodoconhecimento.com.br/administration-des- 
organisations. Par conséquent, la gestion des personnes est une fonction de gestion qui couvre la participation des personnes au sein des organisations afin d'atteindre les objectifs et les objectifs organisationnels et individuels souhaités (POCHMANN, 2015; STECCA, 2014).

Pessoa et al. (2017, p. 02), décrit que :

II est important aujourd'hui que les organisations utilisent des outils technologiques pour résoudre le problème de leur entreprise. Avec les progrès technologiques, les outils sont devenus puissants et, dans une certaine mesure, captivants, par la facilité d'utilisation et l'intégration avec divers systèmes. Les professionnels doivent utiliser des outils pour les aider dans la vie quotidienne de l'entreprise.

Pour que l'entreprise ait un bon développement, il est nécessaire que tous les départements soient alignés, avec un responsable actif dans chaque département. Le service de gestion du personnel exerce une grande importance pour une organisation, car à travers lui toute l'équipe de travail est recrutée et sélectionnée, en plus d'impliquer tout ce qui est lié à l'entreprise, que ce soit en interne ou en externe: environnement, politiques, société et concurrents (REIS et al., 2018).

Dans le monde entier, les entreprises recherchent à travers des outils technologiques, un moyen de maximiser leur entreprise, en se concentrant sur leur performance, leur efficacité, la satisfaction de la clientèle, la rentabilité et l'efficacité du service. Dans les systèmes d'entreprise, nous pouvons percevoir que les formes d'évaluation consolident le travail effectué par la gestion des personnes, un concept largement défendu par plusieurs auteurs comme parle Lima et Figueiredo (2020) lors de l'élaboration de la thèse que seule l'évaluation de ce qui a été fait au cours d'un temps donné peut être identifiée la productivité atteinte dans les secteurs.

En ce sens, l'amélioration de la qualité des organisations au cours des dernières décennies grâce aux technologies a apporté des avantages au marché des entreprises, tels que: l'optimisation des services, une meilleure performance des employés, la compétitivité, la maximisation des résultats, entre autres (OLIVEIRA;

RC: 93822

Disponível em: https://www.nucleodoconhecimento.com.br/administration-des- 
VASCONCELOS, 2005). Dans ce contexte, la gouvernance d'entreprise est devenue la structure qui vise à assurer les décisions relatives à l'organisation, garantissant ainsi les intérêts des parties prenantes (BARROS; DA SILVA, GOESE, 2015).

La gouvernance informatique a apporté de l'innovation à la gestion des personnes. Novato (2014) mentionne que la gouvernance informatique a été classée comme étant un ensemble d'éléments qui apportent des pratiques, des normes et des relations structurées, qui sont dirigées par les gestionnaires et les techniciens informatiques, visant à assurer des contrôles organisationnels, à élargir les processus de performance et de sécurité, à minimiser les risques et à optimiser l'application des ressources, ce qui amène l'informatique dans un alignement axé sur l'entreprise.

Par conséquent, les nouvelles technologies suggèrent des transformations non seulement dans les processus d'affaires, mais aussi dans la façon dont les produits et services sont créés et commercialisés, la structure et les objectifs de l'entreprise, la dynamique de la concurrence et la nature même de l'entreprise et de ses employés (TIDD; BESSANTE, 2015; KON, 2016).

Ainsi, la gouvernance informatique a été soulignée comme un outil efficace et sûr dans la gestion des personnes pour la mise en forme de la structure organisationnelle, impliquant la définition de personnes pour atteindre le cadre professionnel de l'organisation, afin qu'une division de travail harmonique puisse être développée par cette équipe avec des responsabilités visant en commun la croissance de l'entreprise (LORENZETTI et al., 2014).

\subsection{RÉSEAUX SOCIAUX POUR LE RECRUTEMENT ET LA SÉLECTION D'UNE ENTREPRISE}

Lorsqu'on fait référence aux technologies de l'information et de la communication au recrutement de personnes, on dit que les technologies ont facilité la vie de

RC: 93822

Disponível em: https://www.nucleodoconhecimento.com.br/administration-des- 
nombreuses organisations, aidant à la sélection de bons professionnels. Le potentiel des technologies, pour un bon recrutement et une bonne sélection, est de plus en plus utilisé. Dos Santos; De Oliveira et Centurión (2018) soulignent que les technologies émergent comme des alliés dans le recrutement et la sélection du personnel. Grâce à des moyens technologiques, il est possible que les gens puissent chercher un emploi et être sélectionnés.

L'utilisation des réseaux sociaux pour la sélection du programme selon Lago; Da Silva e Barbosa (2018) devient de plus en plus réel, car, de l'utilisation des réseaux sociaux, il y a un plus grand nombre de personnes pour la sélection du poste offert, ce qui permet de trouver l'employé idéal pour le poste souhaité dans l'entreprise.

Queiróz et Sanches (2019, p. 472) mentionnent que :

Parallèlement aux technologies, des réseaux sociaux ont vu le jour, où il est possible de prendre conscience de la vie personnelle des gens en temps réel; Twitter, Instagram, Facebook, vous pouvez difficilement trouver quelqu'un qui n'a pas au moins un de ces réseaux sociaux ces jours-ci. Pour cette raison, il est courant que, dans les processus de sélection, des réseaux soient utilisés pour établir un profil personnel du candidat en fonction de ses postes, ce qui constitue un critère d'exclusion des candidats.

Dans la perception de Melo (2014), les réseaux sociaux ont d'abord été considérés comme des instruments de rencontres et de retrouvailles dans une société basée sur la distance entre les personnes due à la mondialisation. Cependant, le présent permet aux réseaux sociaux non seulement d'être à ces fins, mais d'étendre leurs conceptions et d'évoluer permettant la connaissance et le contact entre des personnes étranges avec des groupes d'intérêt spécifiques.

Un exemple d'utilisation des réseaux sociaux est observé dans le recrutement online. Le recrutement en ligne est un outil qui diffère du recrutement traditionnel parce qu'il s'agit d'une technique utilisée, associée à des technologies telles qu'Internet. Ce

$\mathrm{RC}: 93822$

Disponível em: https://www.nucleodoconhecimento.com.br/administration-des- 
type de recrutement vise à divulguer les offres d'emploi et à sélectionner les personnes uniquement via des réseaux virtuels (DE LIMA: RABELO, 2018).

Pedroso (2016, p. 25), décrit que :

Le recrutement en ligne est une méthode en pleine croissance, car de plus en plus d'individus mettent leur Curriculum Vitae (CV) à disposition sur le marché en ligne parce qu'ils reconnaissent leur potentiel. Le succès de cette méthode est intrinsèquement lié au fait que les entreprises peuvent atteindre un plus grand bassin de candidats ayant du potentiel, car les restrictions géographiques sont moins importantes, c'est-à-dire que les individus peuvent être approchés par des entreprises de n'importe où dans le monde ainsi que peuvent postuler à un emploi de n'importe quelle région du monde.

Cassiano; Lima et Zupani (2016) identifient que la façon dont la diffusion sur les réseaux sociaux de la fréquence du recrutement se produit est considérée comme passive. Cette forme de recrutement a lieu à partir de la diffusion de l'employé sur les médias sociaux, l'un de ces médias concerne Facebook et Instagram. Un autre recrutement passif implique le réseau social de linkedin, dans ce réseau social, le candidat est sélectionné par sa référence de profil dans laquelle ses qualifications apparaissent. II est à noter que ces recrutements sont effectués par les réseaux sociaux et que chaque réseau social apporte une caractéristique différenciée sur le candidat, il appartient donc au recruteur de savoir s'ils sont directs avant le choix de ses candidats pour la sélection.

De Lima et Rabelo (2018, p. 06) déclarent que :

Le recrutement électronique peut également fournir des informations constantes aux candidats concernant les opportunités d'emploi de différents domaines et organisations. Le recrutement traditionnel est souvent publié dans des annonces de journaux et de magazines qui nécessitent un nombre limité de caractères ou d'informations. En ce sens, le recrutement en ligne peut être considéré comme une source de clarté et aussi un moyen dynamique, car on peut diffuser des liens qui dirigent les candidats vers des contenus pertinents sur l'organisation.

RC: 93822

Disponível em: https://www.nucleodoconhecimento.com.br/administration-des- 
Lago; Da Silva Manhães et Barbosa (2018) mentionnent dans leurs recherches que les réseaux sociaux et les médias optimisent le temps du recruteur et la sélection des personnes. L'utilisation d'outils technologiques améliore l'approximation entre l'entreprise et le candidat apportant de l'agilité dans la diffusion des offres d'emploi et dans la réception des CV à sélectionner.

Selon Oliveira (2013, p. 38) :

De bonnes relations interpersonnelles sont l'un des facteurs qui influencent la performance satisfaisante des productions quotidiennes d'un groupe. Une équipe qui travaille ensemble pour le même objectif atteint avec qualité et efficacité ce qui est proposé par son leader.

En ce sens, il est essentiel que le gestionnaire connaisse de près les anides et les objectifs de chaque employé pour l'organisation. C'est pour qu'il n'y ait pas d'inconfort juridique lors de l'identification de quelque chose qui ne peut pas apporter d'avantages à l'organisation, ou même au contraire, l'employé est la cible de quelque chose d'indésirable.

\section{CONSIDÉRATIONS FINALES}

Les recherches effectuées ont soulevé un sujet pertinent sur le recrutement et la sélection dans les organisations dans le cadre des changements résultant de la technologie. La gestion des personnes est un secteur dans lequel l'ensemble du processus de ressources humaines se développe, dans cet environnement, la recherche de l'employé idéal commence.

Compte tenu des changements de la mondialisation, le recrutement et la sélection des personnes ont été marqués par de nouvelles techniques de choix, parmi ces nouvelles techniques qui étaient jusqu'alors face à face, les réseaux sociaux gagnent de l'espace, abattant les obstacles géographiques et élargissant la concurrence pour le poste vacant prévu.

RC: 93822

Disponível em: https://www.nucleodoconhecimento.com.br/administration-des- 
II devient évident que l'utilisation des technologies assume un rôle pertinent dans l'embauche de cet employé, à travers les réseaux sociaux, un outil potentiel de recrutement et de sélection, font des technologies un allié dans la prise de décision.

Facebook, Instagram, Twitter, Linkedin et d'autres réseaux sociaux ont été remarqués par les recruteurs afin de trouver le candidat idéal. Cependant, chaque réseau social a sa caractéristique et que le recruteur doit savoir cibler chaque réseau social à la recherche du candidat idéal pour son entreprise.

Il est donc conclu que les réseaux sociaux sont des outils technologiques qui aident à la gestion des personnes à la recherche de leurs candidats. II est souligné l'importance d'étendre cette étude sur la base de preuves scientifiques, dans laquelle des recherches peuvent être menées au sein des entreprises qui utilisent les réseaux sociaux pour surveiller leurs employés.

\section{RÉFÉRENCES}

\section{ALBERTON, Luiz. Uma contribuição para a formação de auditores contábeis}

independentes na perspectiva comportamental. 2002. 270 f. Monografia (Programa de Pós-Graduação em Engenharia de Produção). Universidade Federal de Santa Catarina. 2002.

BARROS, Claudio Marcelo Edwards; DA SILVA, Pedro Ylunga Costa; VOESE, Simone Bernandes. Relação entre o custo da dívida de financiamentos e governança corporativa no Brasil. Journal of Accounting, Management and Governance, v. 18, n. 2, 2015.

CANALLI, Tiago; DE ANDRADE, Érica Fernanda Pereira. Recrutamento interno: uma nova perspectiva sobre plano de carreira. Revista Eletrônica Organizações e Sociedade, 2016, 5.3: 88-100.

$\mathrm{RC}: 93822$

Disponível em: https://www.nucleodoconhecimento.com.br/administration-des- 
CARVALHO, leda Maria Vecchioni. Recrutamento e seleção por competências. Rio de Janeiro: Editora FGV, 2015.

CASSIANO, Cecilia Nascimento; LIMA, Luciana Campos; DOS SANTOS ZUPPANI, Tatiani. A eficiência das redes sociais em processos de recrutamento organizacional. NAVUS-Revista de Gestão e Tecnologia, v. 6, n. 2, p. 52-67, 2016.

CHIAVENATO, Idalberto. Recursos humanos.7 ed. São Paulo: Atlas, 2002.

COSTA, Esdras da Silva et al. Análise das relações e ações conjuntas entre as empresas do APL têxtil da região metropolitana de São Paulo: contribuições para o seu crescimento. Interações (Campo Grande), v. 19, p. 401-415, 2018.

DE BEM NORO, Greice; STÜKER, Camila; DE OLIVEIRA, João Helvio Righi. A relação das características inerentes aos perfis de liderança no desenvolvimento do processo coaching. Revista Capital Científico-Eletrônica (RCCe) - ISSN 21774153, v. 13, n. 1, p. 59-76, 2015.

DE LIMA, Aryane Santos Henriques; RABELO, Aline Andrade. A importância do erecrutamento e seleção online no processo organizacional. Revista Psicologia,Diversidade e Saúde, v. 7, n. 1, p. 139-148, 2018.

DOS SANTOS, Márcia Gomes; DE OLIVEIRA, Rodrigo Cesar Reis; CENTURIÓN, Wanusa Campos. Recrutamento e seleção estratégicos: processos tradicionais e a influência das mídias sociais. Ideias e Inovação-Lato Sensu, v. 4, n. 3, p. 57, 2018.

KON, Anita. Ecossistemas de inovação: a natureza da inovação em serviços. Revista de Administração, Contabilidade e Economia da Fundace, v. 7, n. 1, 2016.

LAGO, Barbara; DA SILVA MANHÃES, Ana Claudia Tavares; BARBOSA, Marcus Vinicius. O uso de novas tecnologias digitais no recrutamento e seleção de

RC: 93822

Disponível em: https://www.nucleodoconhecimento.com.br/administration-des- 
talentos. Revista Interdisciplinar em Gestão, Tecnologia e Saúde, v. 1, n. 01, p. 161-178, 2018.

LEAL, Débora Ribas; MATTOS, Gisele Domingues de; FONTANA, Rosane Teresinha. Trabalhador com deficiência física: fragilidades e agravos autorreferidos. Revista brasileira de enfermagem, v. 66, n. 1, p. 59-66, 2013.

LEITE, André Mourão. O impacto da transformação digital na gestão de pessoas: abordagens estratégicas para atrair, reter e motivar talentos da área de TI na região da grande Florianópolis. Gestão de pessoas-Unisul Virtual, 2019.

LIMA, Ricardo Leite; FIGUEIREDO, Giane Lourdes Alves de Souza. Novas estratégias do marketing 4.0 para as organizações. Facit Business and Technology Journal, v. 1, n. 21, 2020.

LORENZETTI, J. et al. Organização do trabalho da enfermagem hospitalar: abordagens na literatura. Texto Contexto Enferm., Florianópolis, v.23, n. 4, p. 110412, out-dez, 2014.

MELO, Cristiane Maria Freitas de. Freedom of expression on social networks: right to criticism of the employee versus image and honor of the employer. 2014. 136 f. Dissertação (Mestrado em Direito) - Pontifícia Universidade Católica de São Paulo, São Paulo, 2014.

MOTTA, Marlton Fontes, et al. O Uso das Redes Sociais no Ambiente de Trabalho e as Estratégias da Gestão de Pessoas para a Gestão de Resultados. In: Congresso de Gestão, Negócios e Tecnologia da Informação-CONGENTI. 2017.

MOZER, André Emílio. Recrutamento e seleção-uma proposta para recrutamento e seleção interno. Gestão em Foco: Revista Eletrônica On Line. Amparo: SP, 2016, 8.1.

RC: 93822

Disponível em: https://www.nucleodoconhecimento.com.br/administration-des- 
NOVATO, Douglas. 0 que é Governança de TI? OFICINA DA NET. 2014.Disponível em: https://www.oficinadanet.com.br/post/12712-o-que-egovernanca-de- Acesso em: 13/mai/2021

OLIVEIRA, Camila Miles; BUENO, Rose Ângela Vieira Passos. Uma análise da contribuição dos testes psicológicos no processo de recrutamento e seleção de pessoal. Simpósio de Produções Acadêmicas em Psicologia do Univag, n. 1, 2018.

OLIVEIRA, Rosane S. O papel do gestor na motivação dos seus colaboradores. Cairu em Revista. Jan 2013, Ano 02, n02, p. 33-41, ISSN 22377719.

PEDROSO, Rita Cipriano. Redes sociais e recrutamento. 2016. $81 \mathrm{f}$. Tese de Doutorado. Universidade Católica de Portugal. Porto, Portugal, 2016.

PESSOA, Cláudio Roberto Magalhães et al. Da gestão de TI à gestão de informação e tecnologia: uma abordagem teórica da evolução do conceito= From management of technology and information to a management of information and technology: a theoretical approach of concept development. Descobrimentos da Ciência da Informação: desafios da Multi, Inter e Transdisciplinaridade (MIT): XVII Encontro Nacional de Pesquisa em Ciência da Informação (ENANCIB), 2016.

POCHMANN, Márcio. O emprego na globalização: a nova divisão internacional do trabalho e os caminhos que o Brasil escolheu. Bomtempo Editorial, 2015.

PORTO, Daniel da Silva et al. Gestão de pessoas: o caso da empresa Yes Contact Center no Município de Florianópolis/SC-Brasil. 2016. Dissertação de Mestrado.

QUEIRÓZ, Monique Mazon; SANCHES, Nathalia Paes. As influências das redes sociais no direito do trabalho. Revista do Tribunal Regional do Trabalho da 15a Região, n. 55, p. 271-280. 2019

$\mathrm{RC}: 93822$

Disponível em: https://www.nucleodoconhecimento.com.br/administration-des- 
REIS, Thompson Augusto, et al. Endomarketing, Liderança e Comunicação: reflexos na organização. Caderno Profissional de Marketing-UNIMEP, 2018, 6.1: 1-17.

RIBEIRO, Antonio de Lima. Gestão de pessoas. Saraiva Educação SA, 2017.

SANTOS, Suênia Soares. Recrutamento e seleção: um estudo do impacto da tecnologia praticado por hotéis da orla de João Pessoa-PB. 2018. $44 \mathrm{f}$. Monografia (Graduação em Hotelaria). Universidade Federal da Paraíba. Paraíba. 2018.

SILVA, Bruno de Oliveira Fernandes da, et al. Teoria das Organizações. 2020. 46 f. USP - Universidade de São Paulo. Faculdade de Economia, Administração e Contabilidade - FEA-RP. São Paulo. 2020.

SILVA, Jessyca Rodrigues Henrique da. Impactos da tecnologia nos processos de gestão de pessoas: um estudo no setor hoteleiro. 2019. 129f. Dissertação (Mestrado em Turismo) - Centro de Ciências Sociais Aplicadas, Universidade Federal do Rio Grande do Norte, Natal, 2019.

SILVA, Vanessa; FERRETT, Kézia; MANCINI, Ronaldo. Recrutamento e Seleção: Um estudo sobre as técnicas utilizadas nas organizações. ETIC-ENCONTRO DE INICIAÇÃO CIENTÍFICA, 10.10. 2014.

SPADER, Gabriele A influência da competência profissional, da inteligência emocional, da produtividade e do engajamento sobre a satisfação com o trabalho em um ambiente de serviços de saúde. 2019. 93 f. Dissertação (Mestrado em Administração). Universidade de Caxias do Sul. 2019.

STECCA, Jaime Peixoto. Estratégias genéricas na gestão de pessoas e comprometimento organizacional: um estudo em sociedades cooperativas de crédito. 2014. 144 f. Tese. (Doutorado em Administração, Economia e Contabilidade). Universidade de São Paulo. 2014

RC: 93822

Disponível em: https://www.nucleodoconhecimento.com.br/administration-des- 
TIDD, Joe; BESSANT, Joe. Gestão da inovação-5. Bookman Editora, 2015.

WANZERLLER, Rafaela et al. Gestão de pessoas: visão estratégica sobre treinamento e desenvolvimento no contexto das organizações do século XXI. Revista de Administração e Negócios da Amazônia, v. 10, n. 2, p. 67-84, 2018.

Soumis : Mai 2021.

Approuvé : Juillet 2021.

RC: 93822

Disponível em: https://www.nucleodoconhecimento.com.br/administration-desaffaires/collaborateurs 